\section{More data needed on scientific misconduct}

Donald Kornfeld and Sandra

Titus contend that implementing known remedies for scientific misconduct is better than calling for further research (Nature 548, $31 ; 2017)$. I disagree: we need research to determine whether such measures are working.

Just as the presence of more police officers leads to more arrests, the expansion of efforts to counter scientific misconduct is exposing more cases. Institutions already implement punitive measures, operate offices for research integrity and run ombudsman systems. Journals retract papers more frequently than they used to, and scandalous cases are widely publicized online (see F. Hesselmann et al. Curr. Sociol. 65, 814-845; 2017). Prevention tactics include setting up university commissions for good scientific practice, and introducing regulations for responsible research.

Still, the detection of more cases will stimulate calls for ever-harsher countermeasures. However, criminological research has shown that such law-andorder policies have devastating effects - for example, by discriminating against people who are already disadvantaged (see M. Cavadino and J. Dignan The Penal System; Sage, 2013). Although such policies have political appeal, they can be ineffective and unjust.

What is needed is a measured response to the problem of scientific misconduct, which can stem only from more research. Martin Reinhart Humboldt University of Berlin, Germany. martin.reinhart@hu-berlin.de

\section{NOAA's strategy for unified modelling}

As Earth's systems change at an unprecedented rate, it is becoming harder to model seemingly unconnected phenomena such as atmospheric processes and coastal-zone economies. Tight budget constraints make the situation worse. The US National Oceanic and Atmospheric Administration (NOAA) has therefore developed a unified modelling strategy that will work for the entire agency.

Unified modelling provides a common framework for interoperability across disciplines (even if they are using different information) by applying the appropriate number of models to a similar set of tasks and requirements (see go.nature.com/2vmzhqo). For example, the modelling enables seamless integration of weather-to-climate predictions - crucial for modernizing the US National Weather Service's operational models. It also allows the projection of productivities for fisheries under different climate-change scenarios. The breadth of this applicability, from research to operations, will benefit NOAA's partners and the public.

Our unified approach will also optimize modelling costs and resources. It will help to achieve a critical integrated mass for improving the NOAA modelling enterprise as it moves towards prediction of the full Earth system.

Jason S. Link NOAA National Marine Fisheries Service, Woods Hole, Massachusetts, USA.

Hendrik L. Tolman NOAA National Weather Service, Silver Spring, Maryland, USA.

Katelyn Robinson NOAA Office of Oceanic and Atmospheric Research, Silver Spring, Maryland, USA. jason.link@noaa.gov

\section{Turn war rooms into peace rooms}

The resurgence of terms such as 'cold war' and 'clash of cultures' in the media reflects a dangerous social dynamic that could drive societies to the brink of recession, civil war and societal collapse. We suggest that a more modern, open and scientific strategy might help to prevent history from repeating itself.

Today's strategic 'war rooms' use big data, artificial intelligence and cognitive environments to manage conflicts and crises or run big business. Recasting them as 'peace rooms' would be better in tomorrow's world - they would then be more democratic and would operate with greater transparency for legitimacy. This would help to build trust and expose flaws in the system.

Peace rooms could be run by interdisciplinary, international scientific teams to integrate the best available knowledge. They would rely on input from multiple stakeholders — including cities, civil society, non-governmental organizations, citizen scientists and crowdsourcing - to find solutions that work for as many people as possible. The rooms would be supervised by ethics experts to ensure that innovative outcomes are used responsibly.

This is in line with approaches such as democratic capitalism and digital democracy (see also go.nature.com/2vm2gua and D. Helbing and E. Pournaras Nature 527, 33-34; 2015). Peace rooms could change how strategic decisions are made in crisis situations, guiding us from uncontrollable conflict to the sustainable development that the world needs now. Dirk Helbing Swiss Federal Institute of Technology (ETH) Zurich, Zurich, Switzerland. Peter Seele University of Lugano (USI), Lugano, Switzerland. dirk.helbing@gess.ethz.ch D.H. declares competing financial interests; see go.nature.com/2xxkbdi.

\section{Conferencing in 140 characters}

The use of Twitter hashtags to promote conferences and share presented content is on the rise - an example is the World Seabird Twitter conference, now in its third year. Twitter and other socialmedia platforms can boost outreach, engagement and peerto-peer networking beyond conventional conferencing (see also S. D. Silberberg et al. Nature 548, 153-154; 2017).

Twitter accounts that rotate their curators, such as Biotweeps (@biotweeps), are improving science communication (see go.nature.com/2h2wdpu). In June, Biotweeps hosted its first annual conference, featuring 60 presenters from 12 countries covering a range of biological topics. They had just 15-30 minutes to present their tweets. Forced to be creative by the medium's limit of 140 characters, many used GIF images and infographics.

Conference tweets were collected by the hashtag \#BTCon17. More than 1,200 people responded worldwide (with a theoretical reach of some 22 million followers). By contrast, 573 participants tweeted at an international urology conference that was promoted on social media (S. E. Wilkinson et al. BJU Int. 115, 839-846; 2015). According to a hashtag report (talkwalker.com), the Biotweeps conference resulted in 9,500 engagements with conference tweets.

Anthony Caravaggi University College Cork, Cork, Ireland. Katherine James Newcastle University, UK. anthony.caravaggi@ucc.ie

CONTRIBUTIONS

Correspondence may be submitted to correspondence@nature. com after consulting the author guidelines at http://go.nature.com/ cmchno. Alternatively, readers may comment online: www.nature.com/ nature. 